\title{
Effect of playing soccer on stress, sociality, and physical fitness in alienated youth: a retrospective study
}

\author{
Sunhee Park ${ }^{1,2}$, Jaewan Park ${ }^{2,3}$, Jaehyun Yoo ${ }^{4}$, Yong-Seok Jee ${ }^{2, *}$ \\ 'Department of Physical Education, Chungbuk National University, Chungju, Korea \\ ${ }^{2}$ Research Institute of Sports and Industry Science, Hanseo University, Seosan, Korea \\ ${ }^{3}$ Department of Physical Education, Korea University, Seoul, Korea \\ ${ }^{4}$ Department of Health and Human Performance, Sahmyook University, Seoul, Korea
}

This study was to analyze bullying experiences, physical fitness, and stress and social support in young students who have taken part in a soccer program for approximately 3 years. Fifty-two participants who were divided into two groups: a control group that had not experienced alienation (NAG; $n=45,86.5 \%$ ) and an experimental group that had experienced alienation ( $A G ; n=7,13.5 \%$ ). The frequency of alienation in AG was '1-2 times' or 'a few times' per week. NAG believed their involvement in the soccer program helped them improve their relationships with friends, cope with alienation, and overcome bullying. Muscle mass and basal metabolic rate of NAG showed higher levels than those of $A G$. Strength of NAG was significantly higher than that of $A G$. Stress levels of NAG were significantly lower than those of AG. Although social support was not significantly different between groups, there was a higher tendency in NAG to have stronger social support compared to AG. In conclusion, this study suggests that physical fitness and stress levels can be improved by playing soccer. In particular, participating in a soccer program for an extended period of time can benefit individuals who are socially alienated.

Keywords: Alienation, Physical fitness, Stress, Social support, Soccer

\section{INTRODUCTION}

Childhood is a period when friendships are made and social groups are formed. Proper formation of relationships within the peer group has a great influence on the cognitive or emotional development process (Schwartz and Proctor, 2000). For youngsters, parents are an important part of the family, but in order to grow as a member of society, peers cannot be overlooked (Park et al., 2017). During this period when relationships with peers are formed on the basis of trust, students can experience hardships from being alienated by their peers. This can result in not wanting to attend school or avoiding interactions with other students. In particular, bullying leaves scars both physically and mentally, and may cause severe problems not only in the formation of individual personalities, but over an entire lifespan (Le et al., 2017). Being the victim of bullying can cause severe stress and can even lead to suicide. However,

this can be difficult to resolve because many students do not consider the seriousness of bullying or alienation.

Interpersonal relationship stress, which is similar to the stress experienced through bullying, starts off with parents and family being the main subjects, then after reaching school age, can occur in students who begin to form relationships with peers and teachers (Park et al., 2017). In many cases, such stress has a negative influence, and may even have a critical influence on growth and development (Baldwin et al., 2016). To address these problems, both government and civilian organizations are offering several alternatives. Of these, most organizations aim to lower stress levels and minimize bullying through sports programs. Playing sports during childhood can physically, mentally, and socially offer the complete experience of personal activity, help in maintaining a healthy body, and act in resolving any frustration (Ginsburg et al., 2007). In other words, participating in a sports program during

${ }^{*}$ Corresponding author: Yong-Seok Jee iD https://orcid.org/0000-0001-6797-0843 Research Institute of Sports and Industry Science, Hanseo University,

46 Hanseo 1-ro, Haemi-myeon, Seosan 31962, Korea

E-mail: jeeys@hanseo.ac.kr

Received: February 2, 2020 / Accepted: March 7, 2020 
this period improves adaptability to groups, helps develop mental maturity, and builds social skills. In particular, negative emotions such as anxiety, stress, and aggression can be resolved by taking part in physical activity that builds self-confidence and provides a sense of accomplishment from overcoming challenges. Also, through group sports, self-centeredness is lessened so that more meaningful social relationships can be made. Through group activities, collaborative and competitive relationships are formed, respect for other people's opinions are developed, and the basic principles of rules, manners, collaboration, competition, and leadership are learned (Raabe et al., 2019), thereby developing social skills.

Due to the popularity of soccer, there are numerous youth soccer clubs, which is an example of how group sports activities with peers can help develop leadership skills, social skills, and physical fitness. However, few studies have been done on how playing soccer can be beneficial for individuals struggling with interpersonal relationships, bullying, and the lack of social support. In particular, the degree of social support or stress levels are sometimes thought to be linked to physical build or fitness, but there has been no conclusive answer. This study outlines two major hypotheses: (a) Higher physical fitness levels will result in lower stress levels. (b) Higher physical fitness levels will result in less alienation.

\section{MATERIALS AND METHODS}

\section{Subjects}

The study sample consisted of 59 male children who were mem- bers in a youth soccer team. Among them, three were excluded due to a lack of sufficient time spent as a member in a youth soccer team. As shown in Fig. 1, the participants were divided into two groups according to the results of the questionnaire: a control group that did not experience alienation (NAG) and an experimental group that experienced alienation (AG). During allocation phase, two of control group were disqualified since they did not take part in assessment. In the follow-up phase, there were also two subjects of control group who dropped out due to personal reasons. Finally, 45 of control group and seven of experimental group were analyzed in this study. Complete subject characteristics are illustrated in Table 1.

\section{Experimental protocol}

This is a cross-sectional retrospective study, which takes playing soccer as the independent variable and stress, sociality and physical fitness as the dependent variables. All the procedures were explained in detail to all of the participants before they signed an informed

Table 1. Physical characteristics of the subjects

\begin{tabular}{lcrcc}
\hline Variable & $\mathrm{NAG}(\mathrm{n}=45)$ & \multicolumn{1}{c}{$\mathrm{AG}(\mathrm{n}=7)$} & \multicolumn{1}{c}{$Z$} & $P$-value \\
\hline Age $(\mathrm{yr})$ & $10.16 \pm 1.55$ & $9.00 \pm 1.15$ & -1.857 & 0.068 \\
Height $(\mathrm{cm})$ & $139.31 \pm 13.23$ & $130.29 \pm 8.01$ & -1.598 & 0.115 \\
Weight $(\mathrm{kg})$ & $37.84 \pm 13.14$ & $30.51 \pm 8.65$ & -1.408 & 0.167 \\
Soccer carrier (yr) & $2.23 \pm 0.91$ & $2.00 \pm 0.71$ & -0.561 & 0.605
\end{tabular}

Values are presented as mean \pm standard deviation.

$N A G$, group without experiencing alienation; AG, group with experiencing alienation.

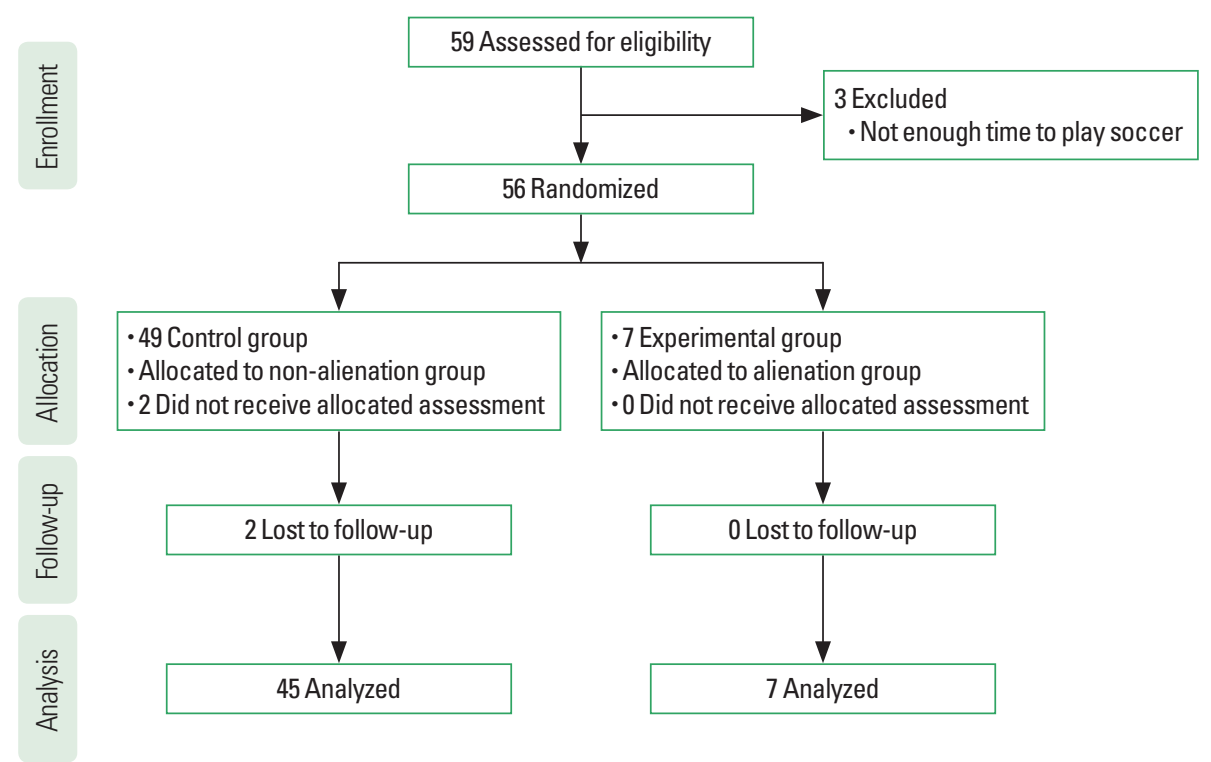

Fig. 1. Flow diagram participants (assessed for eligibility; $n=59$ ) 
consent form that was approved by the Sahmyook University (21040781-AB-N-01-2016121HR). All participant data was kept confidential and was only used for research purposes. They took part in supervised progressive soccer programs as follows. The first phase began with 15 min of warm-up exercises and standing stretches. Standing stretches were performed for $10 \mathrm{~min}$ in order to protect the joints from hyper-extension and -flexion before the workout phase. The second phase started with a 20 min workout that included kicking, passing, and playing. The subjects then completed 20 min of physical training that consisted of abdominal crunches, push-ups, semisquats, and back extensions. The number of repetitions was increased from 10 to 15 in fixed sets of 3 . The 13-15 of ratings of perceived exertion were used as the weightbearing exercise intensity. In the third phase, the subjects played soccer for $40 \mathrm{~min}$ followed by $10 \mathrm{~min}$ of cool-down exercises and supine stretches.

\section{Measurement}

This study applied and used to previous studies to measure bullying experiences, stress levels, and social support. Four questions related to alienation were extracted and applied from a study by Austin and Joseph (1996). Twenty-two questions associated with interrelationship stress and three questions for examining bullying experience and sports activity were extracted from studies by Dudley et al. (1997) and Haltigan and Vaillancourt (2014). Twenty-four questions for examining bullying and social support were applied from a study by Rigby (2000). Cronbach $\alpha$ was calculated to measure the reliabilities of the questionnaires. Questions associated with physical activity, interrelationship stress, degree of social support, and bullying experience were distributed on an interval from a minimum of 0.704 to a maximum of 0.812 . To measure body composition, it was done with the bioelectrical impedance method. Subjects of both groups were assessed by the body composition test to measure body weight, muscle mass, fat mass, body mass index (BMI), percent fat, and basal metabolic rate (BMR). Body composition measurement was used by InBody 230 (Biospace, Seoul, Korea). Analysis of body composition was measured before dinner and after voiding (Cha et al., 2014).

To measure cardioendurance, subjects performed a step test as they listened to music with a $4 / 4$ beat. The step test was performed in the following order: A 28-cm-high bench step was prepared. The height of the bench was adjusted so that the knee was bent at $90^{\circ}$ when the foot was placed on top. The stepping speed was 1 sec per step. The right foot stepped up, followed by the left foot. Then, the right foot stepped down followed by the left foot.
The duration of the test was 5 min. Five seconds after stopping, the heart rate was measured for $10 \mathrm{sec}$ while the subjects were recovering. The maximum oxygen consumption was estimated to using the equation of Uth et al. (2004).

To measure flexibility, the sit and reach flexion test was performed. It was performed as follows. A ruler $(25 \mathrm{~cm}$ outwards, $40 \mathrm{~cm}$ inwards) attached horizontally to the surface of the test platform was prepared. The subjects sat with their feet $5 \mathrm{~cm}$ apart and their heels against the platform. After taking a deep breath the subjects bent forward while exhaling and extended their fingertips to the furthest point possible. Instructions were given to keep their body position steady with their heads between both arms and to not bounce forward while bending. Instructions were also given to keep their knees fully extended. The greatest value after two measurements was recorded.

To measure muscular strength, it was done through grip strength test using a Smedley dynamometer. The involved muscles included the hand muscles and the flexors of the anterior brachial muscles. It was measured by having the subjects hold the dynamometer so that a right angle was formed by the proximal interphalangeal joint. The width was adjusted accordingly. The arms were placed on the sides in a natural resting position with the dynamometer not touching the body. Both hands were consecutively tested twice with the maximum value being recorded.

The sit-up test was used to measure muscular endurance since it is recognized as an appropriate test for children. The test was conducted as follows. Subjects lied down with their backs against the mat with their feet $30 \mathrm{~cm}$ apart while an assistant held both ankles down. Both hands were locked behind the head and the knees were bent at $90^{\circ}$. When the signal was given, the subjects curled their upper body forward so that their elbows touched their knees. Both elbows had to touch the knees and the back had to touch the floor in order for it to be counted as one sit-up. The total number of sit-ups completed in one minute was recorded.

\section{Statistical analysis}

IBM SPSS ver. 18.0 (IBM Co., Armonk, NY, USA) was used for statistical analysis. All data are reported as mean \pm standard deviation. To ascertain the normality of distribution for the examined variables, the Shapiro-Wilk test was used prior to the comparison of measurements. The Mann-Whitney $U$-test, a nonparametric analysis, was used to evaluate the differences in the characteristics between both groups. NAG versus AG was the betweengroup factor and the significance level was set a priori at $P \leq 0.05$ for all analyses. 
Table 2. Difference of soccer programs between groups

\begin{tabular}{lccc}
\hline Group & Period (yr) & Frequency (day/wk) & Skill level \\
\hline NAG $(n=45)$ & $3.07 \pm 1.86$ & $1.16 \pm 0.37$ & $1.82 \pm 0.58$ \\
AG $(n=7)$ & $2.14 \pm 1.46$ & $1.00 \pm 0.01$ & $2.29 \pm 0.76$ \\
$Z$ & -1.042 & -1.125 & -1.766 \\
$P$-value & 0.343 & 0.511 & 0.128 \\
\hline
\end{tabular}

Values are presented as mean \pm standard deviation.

$N A G$, group without experiencing alienation; AG, group with experiencing alienation.

Table 3. Differences of bullying experiences between groups

\begin{tabular}{lcccc}
\hline Group & $\mathrm{NAG}(\mathrm{n}=45)$ & $\mathrm{AG}(\mathrm{n}=7)$ & $Z$ & $P$-value \\
\hline 01 & $1.00 \pm 0.01$ & $2.43 \pm 0.79$ & -7.126 & 0.001 \\
02 & $1.00 \pm 0.01$ & $1.71 \pm 0.95$ & -4.479 & 0.072 \\
03 & $4.00 \pm 1.65$ & $2.00 \pm 1.00$ & -3.067 & 0.006 \\
04 & $3.44 \pm 1.12$ & $1.29 \pm 0.76$ & -4.147 & 0.001 \\
\hline
\end{tabular}

Values are presented as mean \pm standard deviation.

Q1: Have you ever suffered from bullying by anyone in school over the past year?; 02: Have you ever suffered from being bullied by anyone in school this semester?; 03: What kind of students bully other students?; Q4: Which gender bullied you? $N A G$, group without experiencing alienation; $A G$, group with experiencing alienation.

\section{RESULTS}

\section{Soccer program}

Participants took part in supervised progressive soccer programs for approximately 3 years. The daily activity patterns of most subjects did not change during this study. As seen in Table 2, the NAG and AG participated in a soccer program for $3.07 \pm 1.86$ years and $2.14 \pm 1.46$ years, respectively.

\section{Bullying experiences}

Thirteen point five percent of the participants indicated that they experienced being alienated. Table 3 shows the questionnaire results used to group the subjects into either NAG or AG. Q1 asked 'Have you ever suffered from bullying by anyone in school over the past year?' The most common responses of AG subjects were being alienated ' 1 or 2 times' or 'a few times' per week. The score of AG was significantly higher $(P=0.001)$ than NAG. Q2 asked 'Have you ever suffered from being bullied by anyone in school this semester?' Most of the AG subjects answered Q2 saying that they experienced being alienated 'never' or '1 or 2 times' this semester. The score of AG, however, was not significantly different in that of NAG. Q3 asked 'What kind of students bully other students?' Most of the AG subjects answered Q3 saying that they experienced being alienated by 'a student from another class in
Table 4. Difference of alienation responses between groups after sports activity

\begin{tabular}{lccc}
\hline Group & Confidence $^{\text {a) }}$ & Assistance $^{\mathrm{b})}$ & Overcoming $^{\mathrm{cl}}$ \\
\hline NAG & $1.03 \pm 0.16$ & $1.29 \pm 0.46$ & $1.48 \pm 0.51$ \\
AG & $1.00 \pm 0.01$ & $1.17 \pm 0.41$ & $1.00 \pm 0.01$ \\
$Z$ & -0.387 & -0.616 & -1.746 \\
P-value & 0.937 & 0.644 & 0.154 \\
\hline
\end{tabular}

Values are presented as mean \pm standard deviation.

$N A G$, group without experiencing alienation; $A G$, group with experiencing alienation.

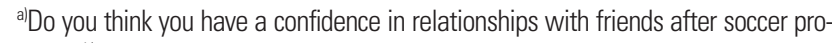
gram? ${ }^{b / D}$ Do you think you have received the assistance does suffer the alienation by a sports activity? ${ }^{\mathrm{c}}$ After being bullied for sporting activities and then did help in overcoming bullying?

the same grade' or 'a senior student in the same school' during this semester. The score of AG was significantly lower $(P=0.006)$ than that of NAG. Lastly, Q4 asked 'Which gender bullied you?' Most AG subjects answered that they experienced being bullied by 'male' students. The score of AG was significantly lower $(P=0.001)$ than that of NAG.

\section{Overcoming bullying experiences}

The differences observed in the three questions between groups are shown in Table 4. The confidence, assistance, and overcoming of alienation were not significantly different between groups. However, a higher tendency was shown in NAG compared to AG. Subjects in NAG considered the soccer program to have improved their relationship confidence with their friends, cope with alienation, and helped to overcome bullying.

\section{Differences in body composition}

As shown in Table 5, although the body weight, fat mass, BMI, and percent fat were not significantly different between groups, the muscle mass $(P=0.028)$ and BMR $(P=0.030)$ of NAG were higher than those of $A G$. These body composition variables may have given a positive effect to subjects who experienced being alienated while also helping to overcome alienation.

\section{Differences in physical fitness levels}

As shown in Table 6, there were no significant differences between groups in flexibility, musculoendurance, and cardioendurance. However, the left grip strength $(P=0.013)$ and right grip strength $(P=0.020)$ of NAG were significantly higher than those of AG. 
Park S, et al. • Soccer improves sociality by enhancing a physical fitness

Table 5. Difference of body composition between groups

\begin{tabular}{lcccccc}
\hline Group & Body weight $(\mathrm{kg})$ & Muscle mass $(\mathrm{kg})$ & Fat mass $(\mathrm{kg})$ & BMl $\left(\mathrm{kg} / \mathrm{m}^{2}\right)$ & Percent fat $(\%)$ & BMR $(\mathrm{kcal})$ \\
\hline NAG & $37.84 \pm 13.14$ & $14.88 \pm 4.56$ & $9.65 \pm 7.04$ & $18.99 \pm 3.67$ & $23.50 \pm 9.33$ & $980.18 \pm 166.33$ \\
AG & $30.51 \pm 8.65$ & $11.57 \pm 1.96$ & $7.61 \pm 5.64$ & $17.71 \pm 3.19$ & $19.91 \pm 13.99$ & $864.71 \pm 72.78$ \\
$Z^{*}$ & -1.408 & -2.299 & -0.670 & -0.872 & -0.657 & -2.301 \\
$P$-value & 0.167 & 0.028 & 0.511 & 0.399 & 0.528 & 0.030 \\
\hline
\end{tabular}

Values are presented as mean \pm standard deviation.

$N A G$, group without experiencing alienation; $A G$, group with experiencing alienation; BMI, body mass index; BMR, basal metabolic rate.

Table 6. Difference of physical fitness levels between groups

\begin{tabular}{lccccc}
\hline Group & $\begin{array}{c}\text { Flexibility } \\
(\mathrm{cm})\end{array}$ & $\begin{array}{c}\text { Musculoendurance } \\
(\text { reps })\end{array}$ & $\begin{array}{c}\text { Cardioendurance } \\
(\mathrm{mL} / \mathrm{kg} / \mathrm{min})\end{array}$ & \multicolumn{2}{c}{ Grip strength $(\mathrm{kg})$} \\
\hline NAG & $7.55 \pm 3.99$ & $27.11 \pm 11.79$ & $64.40 \pm 2.05$ & $19.96 \pm 31.39$ & $15.81 \pm 5.30$ \\
AG & $9.17 \pm 2.88$ & $27.71 \pm 8.32$ & $64.97 \pm 2.07$ & $10.91 \pm 2.16$ & $11.34 \pm 2.46$ \\
$Z^{*}$ & -1.039 & -0.148 & -0.834 & -2.413 & -2.293 \\
$P$-value & 0.315 & 0.896 & 0.414 & 0.013 & 0.020 \\
\hline
\end{tabular}

Values are presented as mean \pm standard deviation.

$N A G$, group without experiencing alienation; $A G$, group with experiencing alienation.

\section{Differences in stress levels}

As shown in Table 7, the stress sum of NAG was significantly $(P=0.010)$ lower than that of AG. More specifically, stress $06(P=$ 0.046), stress $07(P=0.008)$, and stress $09(P=0.003)$ in NAG were significantly lower than those of AG. Stress 06 stated 'I'm stressed because I did not sufficiently share stories with my parents.' Stress 07 stated 'I'm stressed because my parents didn't buy me things I wanted.' Stress 09 stated 'I'm stressed because my friends are bullying me.' The lower stress level of subjects with no experience being alienated showed a positive effect from being involved in the soccer program.

\section{Differences in social support levels}

As shown in Table 8, there was no significant difference in the total amount of social support of NAG compared to AG. However, support $08(P=0.049)$ and support $12(P=0.019)$ were significantly lower in NAG than AG. Support 08 states 'My friends seem to understand me well.' Support 12 states 'My family shares everything with each other.' Finally, although there was a significant difference in the sum scores of social support levels between both groups, there was a higher tendency in NAG compared to AG. This may show that there was a positive effect from participating in the soccer program due to the higher level of support in subjects with no experience of being alienated.
Table 7. Difference of stress levels between groups

\begin{tabular}{lcccc}
\hline Content & NAG & AG & $Z$ & $P$-value \\
\hline Stress 01 & $1.93 \pm 1.01$ & $2.71 \pm 0.95$ & -1.862 & 0.086 \\
Stress 02 & $1.40 \pm 0.72$ & $1.43 \pm 0.79$ & -0.051 & 0.979 \\
Stress 03 & $1.42 \pm 0.69$ & $2.14 \pm 1.35$ & -1.527 & 0.203 \\
Stress 04 & $1.44 \pm 0.76$ & $2.14 \pm 1.35$ & -1.558 & 0.193 \\
Stress 05 & $1.30 \pm 0.63$ & $1.14 \pm 0.38$ & -0.535 & 0.718 \\
Stress 06 & $1.30 \pm 0.59$ & $1.86 \pm 0.69$ & -2.484 & $0.046^{*}$ \\
Stress 07 & $1.47 \pm 0.76$ & $2.57 \pm 0.98$ & -2.978 & $0.008^{*}$ \\
Stress 08 & $1.27 \pm 0.59$ & $1.00 \pm 0.01$ & -1.206 & 0.439 \\
Stress 09 & $1.22 \pm 0.64$ & $2.00 \pm 0.58$ & -3.918 & $0.003^{*}$ \\
Stress 10 & $1.27 \pm 0.72$ & $1.86 \pm 1.21$ & -1.756 & 0.233 \\
Stress 11 & $1.24 \pm 0.65$ & $1.71 \pm 0.95$ & -1.737 & 0.244 \\
Stress 12 & $1.27 \pm 0.65$ & $1.29 \pm 0.49$ & -0.547 & 0.713 \\
Stress 13 & $1.41 \pm 0.84$ & $1.86 \pm 1.21$ & -1.100 & 0.397 \\
Stress 14 & $1.29 \pm 0.69$ & $1.71 \pm 0.95$ & -1.523 & 0.290 \\
Stress 15 & $1.22 \pm 0.67$ & $1.14 \pm 0.38$ & -0.023 & 1.000 \\
Stress 16 & $1.32 \pm 0.74$ & $1.00 \pm 0.01$ & -1.300 & 0.397 \\
Stress 17 & $1.24 \pm 0.65$ & $1.00 \pm 0.01$ & -1.108 & 0.528 \\
Stress 18 & $1.22 \pm 0.64$ & $1.57 \pm 1.13$ & -1.068 & 0.511 \\
Stress 19 & $1.40 \pm 0.84$ & $2.00 \pm 1.41$ & -1.324 & 0.329 \\
Stress 20 & $1.24 \pm 0.68$ & $1.71 \pm 1.11$ & -1.831 & 0.233 \\
Stress 21 & $1.13 \pm 0.50$ & $1.29 \pm 0.76$ & -0.498 & 0.813 \\
Stress 22 & $1.18 \pm 0.58$ & $1.00 \pm 0.01$ & -0.917 & 0.655 \\
Sum & $29.04 \pm 10.23$ & $36.00 \pm 7.37$ & -2.522 & 0.010 \\
\hline Ver &
\end{tabular}

Values are presented as mean \pm standard deviation.

$N A G$, group without experiencing alienation; AG, group with experiencing alienation.

${ }^{*} P<0.05$, significant difference. 
Table 8. Difference of social support levels between groups

\begin{tabular}{lcccc}
\hline Content & $\mathrm{NAG}$ & $\mathrm{AG}$ & $Z$ & $P$-value \\
\hline Support 01 & $3.20 \pm 0.89$ & $3.00 \pm 1.15$ & -0.349 & 0.753 \\
Support 02 & $3.84 \pm 0.56$ & $3.57 \pm 0.79$ & -1.474 & 0.429 \\
Support 03 & $3.42 \pm 0.69$ & $2.71 \pm 1.11$ & -1.803 & 0.103 \\
Support 04 & $3.26 \pm 0.85$ & $2.86 \pm 1.21$ & -0.799 & 0.476 \\
Support 05 & $3.56 \pm 0.81$ & $2.57 \pm 1.40$ & -1.984 & 0.103 \\
Support 06 & $2.89 \pm 0.91$ & $2.57 \pm 1.40$ & -0.478 & 0.674 \\
Support 07 & $3.09 \pm 0.84$ & $2.57 \pm 0.98$ & -1.419 & 0.193 \\
Support 08 & $3.36 \pm 0.74$ & $2.71 \pm 0.76$ & -2.154 & $0.049^{*}$ \\
Support 09 & $3.70 \pm 0.82$ & $3.29 \pm 1.11$ & -1.752 & 0.251 \\
Support 10 & $3.76 \pm 0.57$ & $3.00 \pm 1.41$ & -1.588 & 0.255 \\
Support 11 & $3.70 \pm 0.71$ & $2.86 \pm 1.35$ & -2.168 & 0.100 \\
Support 12 & $3.36 \pm 0.96$ & $2.29 \pm 1.11$ & -2.537 & $0.019 *$ \\
Support 13 & $3.51 \pm 0.89$ & $2.71 \pm 1.11$ & -2.210 & 0.059 \\
Support 14 & $3.64 \pm 0.74$ & $3.14 \pm 1.46$ & -0.548 & 0.694 \\
Support 15 & $3.51 \pm 0.82$ & $3.57 \pm 0.53$ & -0.240 & 0.854 \\
Support 16 & $3.43 \pm 0.87$ & $3.29 \pm 1.11$ & -0.268 & 0.820 \\
Support 17 & $3.18 \pm 0.87$ & $2.50 \pm 1.22$ & -1.449 & 0.179 \\
Support 18 & $3.18 \pm 0.89$ & $3.57 \pm 0.79$ & -1.189 & 0.278 \\
Support 19 & $3.29 \pm 0.97$ & $2.86 \pm 1.35$ & -0.877 & 0.445 \\
Support 20 & $3.56 \pm 0.72$ & $3.50 \pm 0.55$ & -0.595 & 0.637 \\
Support 21 & $3.12 \pm 0.88$ & $3.29 \pm 0.95$ & -0.522 & 0.642 \\
Support 22 & $3.12 \pm 0.86$ & $3.14 \pm 1.21$ & -0.446 & 0.685 \\
Support 23 & $3.42 \pm 0.70$ & $3.71 \pm 0.76$ & -1.301 & 0.258 \\
Support 24 & $2.98 \pm 0.98$ & $3.14 \pm 1.07$ & -0.468 & 0.665 \\
Sum & $79.22 \pm 12.04$ & $71.57 \pm 12.46$ & -1.543 & 0.128 \\
\hline Sum & &
\end{tabular}

Values are presented as mean \pm standard deviation.

$N A G$, group without experiencing alienation; AG, group with experiencing alienation.

${ }^{*} P<0.05$, significant difference.

\section{DISCUSSION}

During childhood, the opportunities to develop social skills can greatly influence their ability to build positive relationships with their peers as they grow older. Therefore, it needs to be determined whether a child is being alienated from a peer group or is the one bullying others (Park et al., 2017). The source of the problem also needs to be understood by determining if it is related to the lack of social support or related to stress. Since recent reports have found that children who are relatively weaker or smaller than their peers are more likely to become victims of bullying, physical characteristics of children should be taken into consideration. To provide possible solutions, it is important to know if participating in sports programs, such as youth soccer programs, can reduce stress caused by interpersonal relationships or alienation. Investigating the physical fitness levels and the physique of children who experience alien- ation is also needed. Lastly, there is a need to find out whether participating in a soccer program can help overcome stress and bullying. The aim of this study was to examine such problems and to provide some possible solutions for bullying youth. The results were as follows. In terms of body composition, there were no significant differences in body weight, fat mass, BMI, and percent fat between groups. However, the muscle mass and BMR in AG were lower than those of NAG. Muscle mass and BMR in AG subjects was generally lower than NAG, thus grip strength, which was used as the parameter for muscular strength, was significantly lower.

Meanwhile, it was expected that the children who did not experience alienation would overcome bullying and build trusting relationships with their friends through participating in the soccer program. These results were similar to those seen in the study by Rodriguez-Ayllon et al. (2019) on the relationship between the participation of children in sports activities and social support and sociability, which showed that for children who participated in sports activities, the more social support they received, the more sociable the child was in terms of responsibility or openness. In particular, the results are also in line with several recent studies, which showed that a lot of athletes formed friendships within the bond of teams, where the athletes have worked towards common goals and trained for success (Dudley et al., 1997; Falcão et al., 2015). The results are also similar to another study which reported that interaction with other peers through the participation in football clubs increases social and environmental satisfaction, physical satisfaction through health promotion, and educational satisfaction through an educational program (García-Calvo et al., 2014; Parnell et al., 2015). The data suggests that subjects reveal concerns about the quality of their own ability, learning, achievement, participation, and social/emotional well-being in the youth sport environment. Similarly, the social support received from both peers and teachers enhanced meaningful engagement with physical education content (Herring et al., 2019). Interpersonal relationships, which shows the ability of a person to maintain good relations and get along well with others, is a concept that binds society's collaborative relationship among groups. By developing interpersonal relationships, stress can be reduced while sociability is improved.

According to the results this study, children who have never experienced alienation had a significantly lower stress sum score than those who have. More specifically, NAG subjects communicated with their parents well (stress \#06), were able to ignore superficial matters (stress \#07), and did not have any friends who bullied them (stress \#09). Namely, after participating in soccer for 3 years, the amount of experience being bullied was minimized with a few 
subjects even stating that playing soccer gave them a positive influence. On the other hand, AG subjects, who have experienced being bullied, showed completely opposite results. Those results were related to low muscular strength and the short amount of time and frequency participating in sports.

The positive effects gained by participating in group sports activities discussed above are not only found in Korea, but also in numerous reports from other countries. Boreham and Riddoch (2001) stated that adequate childhood physical activity provides three main benefits. The first is improved health during childhood. There is mounting evidence that physically active children generally show healthier cardiovascular profiles, have higher peak bone masses, and are leaner than children who are less active. Secondly, there is a biological carryover effect from childhood into adulthood, whereby the physical activity from childhood results in improved adult health status. On the other hand, childhood obesity may act as a precursor for a range of adverse health conditions in adulthood, while higher bone densities in younger people decrease the risk of osteoporosis in later years. Finally, a behavioral carryover effect into adulthood may exist, whereby children who are physically active are more likely to become healthier and active adults.

There are increasing reports that physical activity can improve sociability, reduce stress, and decrease being bullied. Although this study showed that participating in a soccer program reduced stress levels and experiences of being bullied, which is in line with other studies, NAG subjects only indicated a higher tendency in social support (support sum score) than that of AG. This suggests that more specific future studies are needed. From the responses to the social support survey questions (support \#8 and \#12), it is encouraging to see that the subjects felt better understood by their friends and saw a greater connection to their parents after participating in the soccer program.

For a healthy life, it is important to maintain high physical fitness levels and social support, while reducing stress and experiences of being alienated during youth. This study showed that the youth soccer program significantly improved physical strength, reduced stress, and decreased experiences of being bullied. It also appears that the health of the young men who participated in this study was maintained well. This is line with previous research studies that have shown that physical activity can prevent alienation by reducing stress and improving physical fitness. However, some discrepancies exist regarding social support factors. Thus, further investigation into the relationship between physical activity and social support are necessary to prevent alienation. Ultimately, the negative effects of being alienated in young subjects might be re- versed after frequent and prolonged participation in a youth soccer program. However, this study has a limitation as follows. Due to the small sample size of this study, it would be beneficial for future studies to generalize the results with a larger number of subjects.

\section{CONFLICT OF INTEREST}

No potential conflict of interest relevant to this article was reported.

\section{REFERENCES}

Austin S, Joseph S. Assessment of bully/victim problems in 8 to 11 yearolds. Br J Educ Psychol 1996;66(Pt 4):447-456.

Baldwin JR, Arseneault L, Odgers C, Belsky DW, Matthews T, Ambler A, Caspi A, Moffitt TE, Danese A. Childhood bullying victimization and overweight in young adulthood: a cohort study. Psychosom Med 2016; 78:1094-1103.

Boreham C, Riddoch C. The physical activity, fitness and health of children. J Sports Sci 2001;19:915-929.

Cha JY, Kim JH, Hong J, Choi YT, Kim MH, Cho JH, Ko IG, Jee YS. A 12week rehabilitation program improves body composition, pain sensation, and internal/external torques of baseball pitchers with shoulder impingement symptom. J Exerc Rehabil 2014;10:35-44.

Dudley BS, Johnson DW, Johnson RT. Using cooperative learning to enhance the academic and social experiences of freshman student athletes. J Soc Psychol 1997;137:449-459.

Falcão WR, Bloom GA, Loughead TM. Coaches' perceptions of team cohesion in paralympic sports. Adapt Phys Activ Q 2015;32:206-222.

García-Calvo T, Leo FM, Gonzalez-Ponce I, Sánchez-Miguel PA, Mouratidis A, Ntoumanis N. Perceived coach-created and peer-created motivational climates and their associations with team cohesion and athlete satisfaction: evidence from a longitudinal study. J Sports Sci 2014;32: 1738-1750

Ginsburg KR; American Academy of Pediatrics Committee on Communications; American Academy of Pediatrics Committee on Psychosocial Aspects of Child and Family Health. The importance of play in promoting healthy child development and maintaining strong parentchild bonds. Pediatrics 2007;119:182-191.

Haltigan JD, Vaillancourt T. Joint trajectories of bullying and peer victimization across elementary and middle school and associations with symptoms of psychopathology. Dev Psychol 2014;50:2426-2436.

Herring MP, Monroe DC, Gordon BR, Hallgren M, Campbell MJ. Acute exercise effects among young adults with analogue generalized anxi- 
ety disorder. Med Sci Sports Exerc 2019;51:962-969.

Le HT, Nguyen HT, Campbell MA, Gatton ML, Tran NT, Dunne MP. Longitudinal associations between bullying and mental health among adolescents in Vietnam. Int J Public Health 2017;62(Suppl 1):51-61.

Park JW, Park SH, Koo CM, Eun D, Kim KH, Lee CB, Ham JH, Jang JH, Jee YS. Regular physical education class enhances sociality and physical fitness while reducing psychological problems in children of multicultural families. J Exerc Rehabil 2017;13:168-178.

Parnell D, Pringle A, McKenna J, Zwolinsky S, Rutherford Z, Hargreaves J, Trotter L, Rigby M, Richardson D. Reaching older people with PA delivered in football clubs: the reach, adoption and implementation characteristics of the Extra Time Programme. BMC Public Health 2015; 15:220.

Raabe J, Schmidt K, Carl J, Höner O. The effectiveness of autonomy support interventions with physical education teachers and youth sport coaches: a systematic review. J Sport Exerc Psychol 2019 Nov 12:1-11. [Epub]. https://doi.org/10.1123/jsep.2019-0026.
Rigby K. Effects of peer victimization in schools and perceived social support on adolescent well-being. J Adolesc 2000;23:57-68.

Rodriguez-Ayllon M, Cadenas-Sánchez C, Estévez-López F, Muñoz NE, Mora-Gonzalez J, Migueles JH, Molina-García P, Henriksson H, Mena-Molina A, Martínez-Vizcaíno V, Catena A, Löf M, Erickson KI, Lubans DR, Ortega FB, Esteban-Cornejo I. Role of physical activity and sedentary behavior in the mental health of preschoolers, children and adolescents: a systematic review and meta-analysis. Sports Med 2019;49:1383-1410.

Schwartz D, Proctor LJ. Community violence exposure and children's social adjustment in the school peer group: the mediating roles of emotion regulation and social cognition. J Consult Clin Psychol 2000;68: 670-683.

Uth N, Sørensen H, Overgaard K, Pedersen PK. Estimation of VO2max from the ratio between HRmax and HRrest--the heart rate ratio method. Eur J Appl Physiol 2004;91:111-115. 